\title{
LYMPHANGIOMA-LIKE KAPOSI SARCOMA: CHALLENGES IN DIFFERENTIAL DIAGNOSIS
}

\author{
S.R. Georgescu' ${ }^{1}$ A.M. Limbau', M. Tampa ${ }^{1}$, M.D. Tanasescu ${ }^{3}$, S. Zurac ${ }^{2}$, M.I. Popa ${ }^{3}$ \\ 'Dermatology Department, "Carol Davila” University of Medicine and Pharmacy, Bucharest \\ 2 "Colentina" Clinical Hospital, Bucharest \\ 3"Carol Davila" University of Medicine and Pharmacy, Bucharest
}

\begin{abstract}
Background. Kaposi's sarcoma (KS) is a multifocal vascular neoplasia with uncertain histogenesis, characterized by angioproliferative multifocal tumors affecting mainly the skin. The "lymphangioma-like" or "bullous KS" variant is a rare morphologic expression of KS, accounting for less than $5 \%$ of all cases and appearing among all KS epidemiological subtype. This review provides a comprehensive overview of clinical and pathological characteristics of patients with lymphangioma-like Kaposi's sarcoma LLKS.

Methods. We included 93 patients with Kaposi sarcoma, aged between 36 and 90 years; diagnosis was made as a result of the histopathological examination. The surgical excision samples were fixed in $10 \%$ buffered formalin, paraffin embedded and stained with Hematoxylin-Eosin for histopathological examination. Immunohistochemical staining was performed using the following antibodies: CD34, CD31, actin, myoglobin, desmin, cytokeratin and vimentin.

Results. The histological features of LLKS vary considerably from the traditional KS, classic KS areas have been absent from some lymphangioma-like KS. Most of the patients were diagnosed in nodular stage and confirmed by positive immunohistochemical staining. Clinically, each patient presented with violaceous patches, papules or plaques; some of the patients presented with bullous lesions. All tumour cells, including those associated with LLKS foci, showed a strong and diffuse reactivity for anti-HHV-8 LNA-1and anti-CD34. Conclusions. Differential diagnosis of lymphangioma-like Kaposi's sarcoma LLKS with other vascular tumors may be very difficult and a detailed histologic study in combination with immunohistochemistry, such as staining for HHV-8 latent nuclear antigen, is essential for correctly diagnosing lymphangioma-like KS.
\end{abstract}

Keywords: lymphangioma-like Kaposi's sarcoma, differential diagnosis, immunohistochemical staining

\section{INTRODUCTION}

Kaposi's sarcoma (KS) is a multifocal vascular neoplasia with uncertain histogenesis, characterized by angioproliferative multifocal tumors, affecting mainly the skin. Kaposi's sarcoma (KS) was first described by the dermatologist Moritz Kaposi (1837-1902) in 1872. He presented five patients with violaceous cutaneous tumors of the lower extremities, originally described as "idiopathic multiple pigmented sarcoma of the skin", later named Kaposi's sarcoma (KS), now recognized as classic form of KS. (1) In 1994, Chang et al, identified KS associated human herpes virus type 8 (HHV-8). The virus can be found in the blood of patients before lesions of Kaposi's sarcoma develop. (2) Although HHV-8 is present in the vast majority of all types of $\mathrm{KS}$, there is no agreement on the exact mechanism by which HHV-8 results in the development of KS. It has been shown that HHV-8 is necessary but not sufficient for the development of all types of KS. (3)

Along the time, this tumor has received much attention after recognition of its association with the acquired immune deficiency syndrome (AIDS) in the early 1980 s. Nowadays, KS remains the most prevalent malignancy among patients with AIDS.

Four different clinical forms of KS have been described, which are characterized by the distinct patient population affected: classic KS, AIDS-asso- 
ciated epidemic KS, African-endemic KS, and iatrogenic immunosuppressive drug-associated $\mathrm{KS}$, seen predominantly in organ transplant recipients. (4) The classic $K S$ favors the legs of elderly men of Mediterranean origin, with a peak incidence of about 40-70 years. Injuries occurring in the lower extremities then develop slowly towards the proximal areas. The oral mucosa and gastrointestinal tract are rarely involved. (5) Epidemic (AIDS-related) Kaposi's sarcoma develops in people who are infected with HIV. It may occur in the late stage of AIDS, but also as a manifestation of onset in young people, aged between 20-50 years. Involvement of the oral cavity, face, genital mucosa, lungs and gastrointestinal tract (particularly the stomach and duodenum) are common and occurs more often than in classic KS. AIDS-related KS has a variable clinical course and may worsen after a significant decline in the CD4+ cell count. Kaposi sarcoma shows a less aggressive presentation in patients already receiving highly active antiretroviral therapy (HAART). (6)

Immunosuppression-associated $K S$ develops in immunosuppressed persons after an organ transplant or from other autoimmune diseases, treated with immunosuppressive drugs for a long time. Clinical distribution is characterized by localized mucocutaneous or disseminated lesions, with possible visceral involvement, with a clinical course similar to AIDS-related KS. (7) Endemic (African) Kaposi's sarcoma is more common in middle-aged black adults and children from equatorial Africa. This type usually affects the lymph nodes and other organs and can progress quickly. The lymphadenopathic form is aggressive, typically affects lymph nodes rather than the skin. (8)

The diagnosis of KS is confirmed by a skin biopsy of a suspected lesion. Serology for HHV-8 is not required.

In all 4 clinical forms of $\mathrm{KS}$, the lesions go through similar histological phases, from patch to plaque to nodular stages. In early developing KS, during the patch stage the lesions may be oval redbrown macules often following skin lines. The lesion histologically shows reduced proliferation of new small blood vessels around larger dilated vascular spaces and endothelial cells without mitoses. Along adnexal structures and vessels we found small clefts called promontory sign. A perivascular lymphocytic infiltrate is variably observed. In this stage, the lesions are relatively nonspecific. (9) During the plaque stage, the vascular proliferation involves the dermis almost completely; it can extend to the subcutaneous fat. Irregular, vascular clefts filled with erythrocytes, hemosiderin deposits and hyaline globules (PAS-positive globules in tumor cells) and important lymphoplasmacytic infiltrate are all present. In the nodular stage it is also common to find nodular proliferation of spindle cells without nuclear pleomorphism. During this stage, spindle-shaped cells form very small vascular cleft spaces and infiltrate the dermis and subcutaneous tissue. Cytologic atypia, rare mitoses, hemosiderin deposits and hyaline globules can be found. However, some variants are only rarely observed and reported. (10)

The "lymphangioma-like" or "bullous KS" variant is a rare morphologic expression of $\mathrm{KS}$, accounting for less than $5 \%$ of all cases and appearing among all KS epidemiological subtype. (11) The clinical features were first reported in 1957 by Ronchese and Kern. They presented patients who had been previously diagnosed with classic KS, who later developed bullae, with a clear fluid, on their lower extremities. (12) This presentation was later described as "bullous lesions" and was believed to be characteristic of lymphangioma-like KS (LLKS).

The histological findings of the lymphangiomalike variant have been described as the presence of lymphangiectasis without characteristic spindle cell proliferation, bizarre large lacunae and many ectatic vascular dilated channels, usually without erythrocytes, producing bullous eruption. They are located in the upper portion of the dermis. (13) This differs from the telangiectatic variant of $\mathrm{KS}$ in which KS lesions contain large, very congested, ectatic vascular spaces. (14) Lymphangioma-like morphology can occur in patch, plaque or nodular stage lesions and it could be present in all four major clinicopathologic forms of KS patients.

Microscopically, LLKS appears to exist in two forms. The histologic aspect of the first form is similar to a lymphatic tumor, such as a benign lymphangioendothelioma or acquired progressive lymphangioma. This includes a patch or plaque stage lesion in which interanastomosing ectatic vascular channels usually without erythrocytes dissect dermal collagen bands. The promontory sign could be obvious. In the second form, endothelial lined spaces occupy the papillary dermis and the upper reticular dermis, which may produce the pattern to the clinical appearance of „,bullous“ cutaneous lesions. (15)

Clinically, lymphangioma-like KS can present as a typical-appearing $\mathrm{KS}$, but also has a unique clinical appearance termed bulla-like, which is the most common clinical presentation. The formation 
of bulla-like vascular lesions in the lymphangiomalike variant is poorly understood. It is believed that, chronic lymphedema or hemodynamic impairements have been involved. (16) In the literature, although most reported cases of LLKS had a slowly progressive course, it has been reported that lymphangioma-like KS can result in a fatal outcome. (17) The lower extremities are involved in the majority of cases and those are the predilected site of presentation of this KS variant.

\section{METHODS}

We included 93 patients with Kaposi's sarcoma, aged between 36 and 90 years; diagnosis was made as a result of the histopathological examination. The clinical and pathological features of five cases of lymphangioma-like Kaposi's sarcoma (KS) are reported. As with the other subtypes of KS, the lymphangioma-like variant occurs more often in men aged 59-80 years.

The surgical excision samples were fixed in $10 \%$ buffered formalin, paraffin embedded and stained with Hematoxylin-Eosin for histopathological examination. All available hematoxylin and eosin (HE)-stained slides were reviewed. For immunohistochemical studies, paraffin-embedded tissue blocks from all patients were cut at $3-\mu \mathrm{m}$ thick sections, placed on slides, deparaffinized in xylene and hydrated in a decreasing ethanol series. Immunohistochemical staining was performed using the following antibodies: CD34, CD31, actin, myoglobin, desmin, cytokeratin and vimentin.

For immunohistochemical analysis, the presence $(+)$ or absence $(-)$ of immunostaining was determined and the intensity of immunopositivity was classified as strong $(++)$ or weak $(+)$. The distribution pattern (focal or diffuse) as well as the location of stained cells within the tumor arrangement was evaluated.

\section{RESULTS}

The histological features of LLKS vary considerably from the traditional KS, classic KS areas have been absent from some lymphangioma-like KS. Most of the patients, with KS, were diagnosed in nodular stage and confirmed by positive immunohistochemical staining.

Regarding patients with limphangioma-like KS, all of the five LLKS biopsy specimens revealed characteristic light microscopic features of patch/ plaque stage of KS, including, ill-defined fascicles of spindle cells associated with numerous irregu- larly shaped vessels that dissected through dermal collagen. Vascular proliferation surrounding dermal vessels and coursed around adnexal structures and similar aspects of "promontory" sign were observed (Fig. 1). In certain areas, mostly in depth, vascular proliferation is made of capilar vascular structures, with slight protruding endothelium, separated by reduced stroma, with a moderate hemosiderin deposits. At this stage, the presence of siderophages, red blood cell extravasation, and a modest perivascular lymphoplasmacytic infiltrate may be encountered. No bacterial colonies are observed on Giemsa stain.

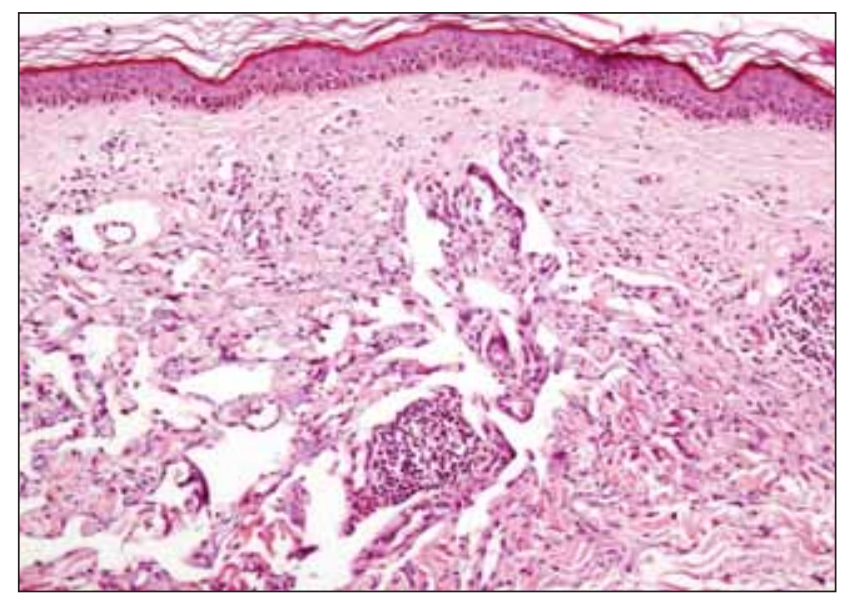

FIGURE 1. KS patch stage, promontory sign: normal dermal structures, partially surrounded by newly formed vessels - it create the impression of protrusion in the tumoral vascular lumen. HE $\times 100$

Immunohistochemical staining was performed using the following antibodies: CD34, CD31, actin, myoglobin, desmin, cytokeratin and vimentin. The histogenesis of KS has been debated and, until now, remains unclear. The endothelial cell is the most widely accepted cell of origin. Several studies have shown that vascular endothelial cell markers like CD31 and factor VIII were present in Kaposi's sarcoma and this supports the origin of KS from blood vessels. On the other hand, another factor as vascular endothelial growth factor receptor-3 (VEGF-3), rather suggest the lymphatic endothelial cell origin. (18) Our sections was stained with anti-CD31 and anti-CD34. CD34 is a less-specific label for endothelial cells than CD31 and tends to show stronger expression in advanced-stage lesions of KS.

The identification and localization of HHV8 within KS lesional cells by using LNA-1 is the most diagnostically helpful immunostaining technique available to differentiate KS from its mimics.

In our study, immunohistochimical tests for the cell type vascular endothelial cell markers (CD31, CD34, FVIII), other mesenchymal cell makers (al- 
pha-smooth muscle actin SMA) and an actin and muscle cell antigen (HHF-35) were tested. All five vascular tumors were positively stained for vascular markers such as CD31, CD34 and factor VIII, which supported their diagnosis (Fig. 2). Proeminent and consistent positivity for CD34 is seen in the spindle cell population. SMA, was totally negative and HHF-35 revealed positivity in non-tumor blood vessels and negativity in vascular proliferation (Fig. 3). Mitotic activity was sparse and no significant inflammatory component was seen. All tu- mor cells, including those associated with LLKS foci, showed a strong and diffuse reactivity for anti-HHV-8 LNA-1 and anti-CD34.

\section{DISCUSSION}

Kaposi's sarcoma presents a large spectrum of clinical and histopathological features. The differential diagnosis can include numerous diseases from benign vascular tumors and inflammatory condition to malignant tumors such as angiosarco-
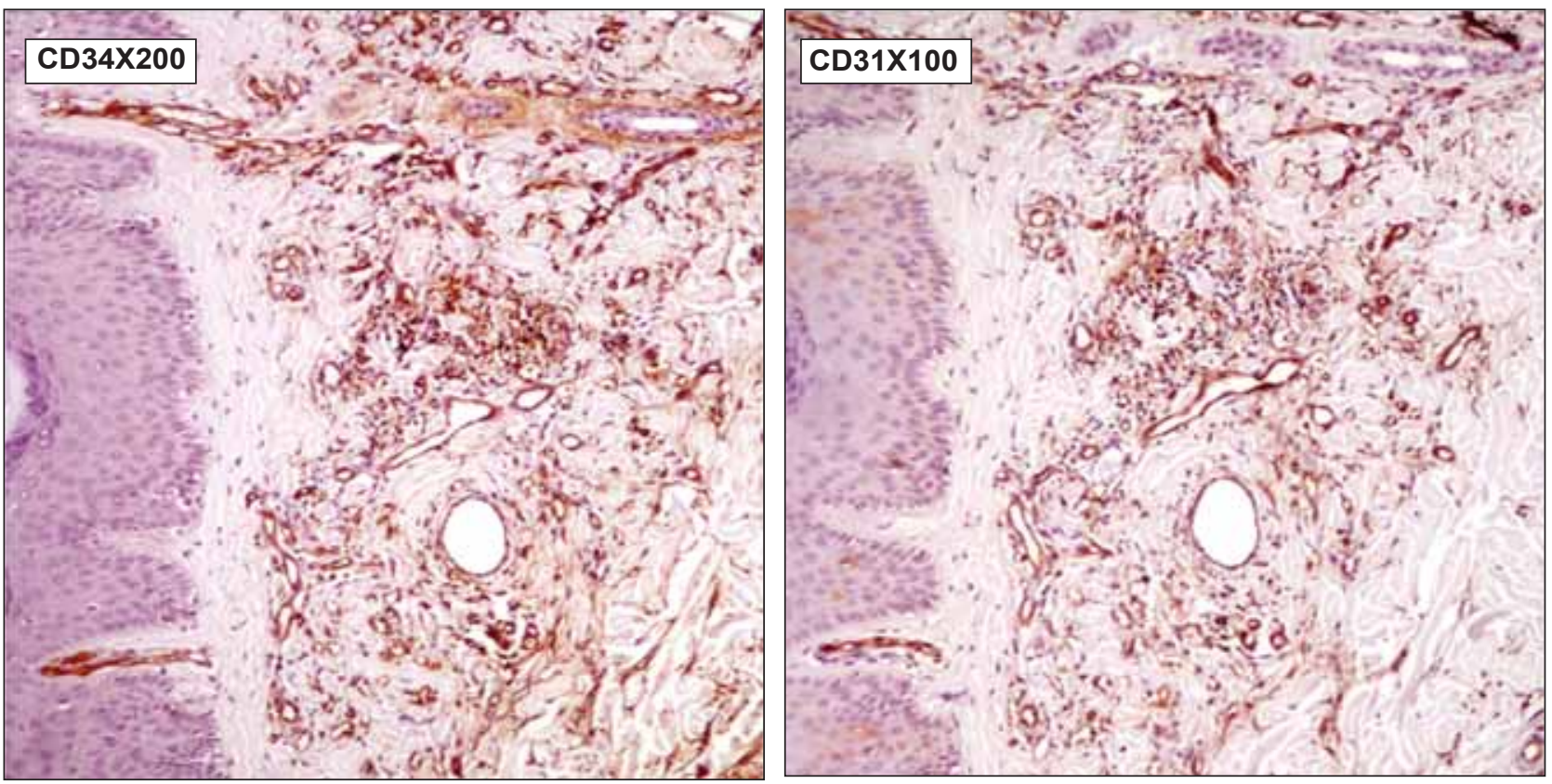

FIGURE 2. KS: Normal and tumoral endothelial cells positive for CD34, CD31
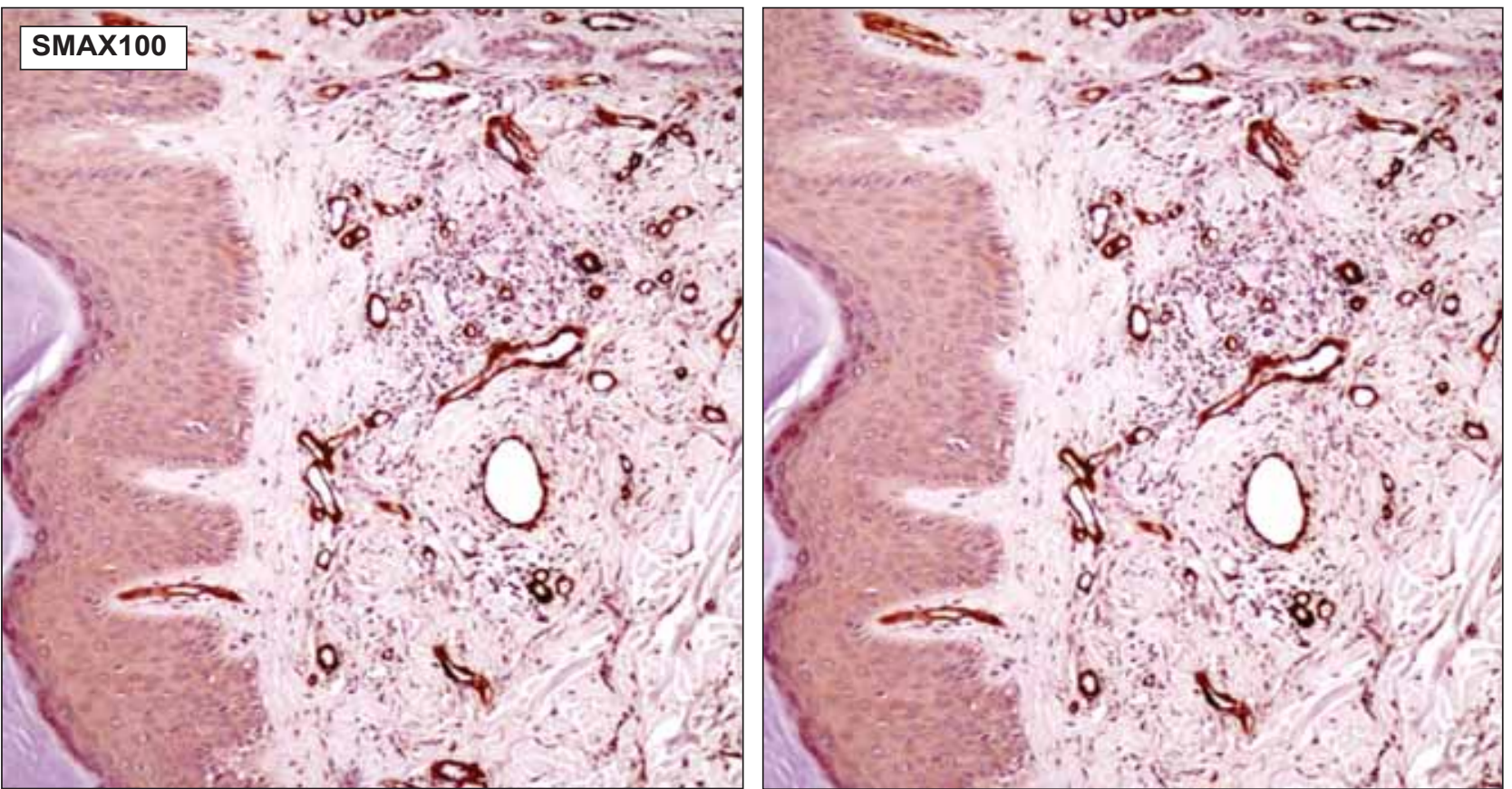

FIGURE 3. KS: SMA negative in the tumor cells and in the periphery of neoplastic vascular structures; positive in normal vascular structures (pericytes) 
ma and from the early macular lesions to late aggressive lesions. (19) Its depend on the histopathological stages, therefore, a precise diagnostic decision making is required in order to manage these patients.

In early macular lesions, differential diagnosis is extremely difficult and it is hard to identify neoplastic origin of vascular lesions. Benign vascular proliferation diseases such as acroangiodermatitis (stasis dermatitis), pyogenic granuloma, early stages of scarring skin lesions, benign lymphangioendothelioma, targetoid hemosiderotic hemangioma or Kaposiform hemangioendothelioma should be distinguished. (20)

The most difficult differential histologic diagnosis of plaque-stage KS includes spindle cell hemangioma. Moderately differentiated angiosarcomas with spindle-cell differentiation and spindle-cell hemangioma are the most important histologic simulants of nodular Kaposi's lesions that may potentially be confused with nodular KS. (21)

In aggressive late-stage lesions, many malignant spindle cell tumors can come into the differential diagnosis especially if clinical details are unavailable. The most important are leiomyosarcoma, malignant peripheral nerve sheath tumors, inflammatory pseudotumor stage II (myoepithelial), dermatofibrosarcoma protuberans, fibrosarcoma and desmoplastic malignant melanoma. (22)

In all the cases, accurate diagnosis may be impossible without an immunohistochemical analysis. CD31 and CD34 are particularly helpful in suggesting a vascular derivation even in poorly differentiated Kaposi's lesions and they can assist in arriving at a correct diagnosis. (23) Clinical history, such as HIV infection or status post-transplant, may strongly support the diagnosis of KS.

\section{CONCLUSIONS}

Lymphangioma-like Kaposi's sarcoma (LLKS) is a rare pathologic variant of Kaposi's sarcoma (KS) that may occur in any of the recognized KS clinical subtypes or as a minor histological component of typical KS. From a clinical point of view, the histologic diagnosis raised some challenging issues with regard to prognosis and treatment. Fortunately, the expanding knowledge of KS biology is assuring increased opportunities for targeted therapies.

As a conclusion the differential diagnosis for lymphangioma-like Kaposi's sarcoma with other vascular tumors remains wide and complex. Nevertheless, it depends on the epidemiological form of the disease, the stage of the disease, as well as the clinical features. Therefore, a detailed histologic study in combination with immunohistochemistry, such as staining for HHV-8 latent nuclear antigen, is essential for correctly diagnosing lymphangioma-like KS.

\section{Acknowledgement}

This paper is partly supported by the Sectorial Operational Programme Human Resources Development (SOPHRD), financed by the European Social Fund and the Romanian Government under the contract number POSDRU 141531.

\section{REFERENCES}

1. Geraminejad P., Memar O., Aronson I., et al. Kaposi's sarcoma and other manifestations of human herpesvirus 8. J Am Acad Dermatol. 2002; 47:641-655

2. Chang Y., Cesarman E., Pessin M.S., et al. Identification of herpesvirus-like DNA sequences in AIDS-associated Kaposi's sarcoma. Science. 1994; 266:1865-1869

3. Ablashi D.V., Chatlynne L.G., Whitman E.J., et al. Spectrum of Kaposi's Sarcoma-Associated Herpesvirus, or Human Herpesvirus 8, Diseases. Clin Microbiol Rev. 2002; 15(3):439-464

4. Uldrick T.S., Whitby D. Update on KSHV-Epidemiology, Kaposi Sarcoma Pathogenesis, and Treatment of Kaposi Sarcoma. Cancer Lett. 2011; 305(2):150-162

5. Iscovich J., Boffetta P., Franceschi S., et al. Classic Kaposi sarcoma: Epidemiology and risk factors. Cancer. 2000; 88:500-517

6. Solivetti F.M., Latini A., Elia F., et al. AIDS-Kaposi Sarcoma and Classic Kaposi Sarcoma: are different ultrasound patterns related to different variants? J Exp Clin Cancer Res. 2011; 30(1):40

7. Firoozan A., Hosseini S.M., Einollahi B., et al. Outcome of Kaposi's sarcoma and graft following discontinuation of immunosuppressive drugs in renal transplant recipients. Transplant Proc. 2005; 37:3061-4

8. Ganem D. KSHV and the pathogenesis of Kaposi sarcoma: listening to human biology and medicine. The Journal of Clinical Investigation. 2010; 120(4):939-949

9. Grayson W., Pantanowitz L. Histological variants of cutaneous Kaposi sarcoma. Diagnostic Pathology. 2008; 3:31

10. Bunn B.K., Carvalho M.V., Louw M., et al. Microscopic diversity in oral Kaposi sarcoma. Oral Surg Oral Med Oral Pathol Oral Radiol. 2013; 115:241-248

11. Ramirez J.A., Laskin W.B., Guitart J. Lymphangioma-like Kaposi sarcoma. J Cutan Pathol. 2005; 32:286-292

12. Ronchese F., Kern A.B. Lymphangioma-like tumors in Kaposi's sarcoma. AMA Arch Derm. 2011; 75(3):418-27

13. Davis D.A., Scott D.M. Lymphangioma-like Kaposi's sarcoma: etiology and literature review. J Am Acad Dermatol. 2000; 43:123-127

14. García M.D., Doval I.G., Cruces M.J., et al. Lymphangioma-like Kaposi sarcoma: Case report. Dermatology Online Journal. 2010; 15(9):13

15. Mohanna S., Sanchez J., Ferrufino J.C., et al. Lymphangioma-like Kaposi's sarcoma: report of four cases and review. J Eur Acad Dermatol Venereol. 2006; 20(8):1010-1 
16. Torchia D., Ketabchi S., Franchi A., et al. "Pseudo-bullous" Kaposi's sarcoma. Eur J Dermatol. 2008; 18(3):359-60

17. Pugalagiri P., Muller S., Kessler H.P., et al. Lymphangioma-like Kaposi sarcoma of the oral mucosa. Oral Surg Oral Med Oral Pathol Oral Radiol. 2013; 116(1):84-90

18. Wolff K., Johnson R.A., Suurmond D. Fitzpatrick Dérmatologie Clinique, 5e édition, Médecine - Sciences Flammarion, Paris. 2005; 536-540

19. Ramirez J.A., Laskin W.B., Guitart J. Lymphangioma-like Kaposi sarcoma. Journal of Cutaneous Pathology. 2005; 32(4):286-292

20. Guillou L., Fletcher C.D. Benign lymphangioendothelioma (acquired progressive lymphangioma) a lesion not to be confused with well-differentiated angiosarcoma and patch stage Kaposi's sarcoma: clinicopathologic analysis of a series. Am J Surg Pathol. 2000; 24:1047-1057

21. Elder D.E., Rosalie E., Bernett L.J., et al. Vascular Tumors: Tumors and Tumor-Like Conditions of Blood Vessels and Lymphatics. Lever's Histopathology of the Skin, 9th Edition. Philadelphia: LippincottRaven. 2005:33-1038

22. Friedman E.R., Farquharson L., Warsch J., et al. LymphangiomaLike Kaposi's Sarcoma Presenting as Gangrene. Rep Oncol Med. 2013

23. Pyakurel P., Pak F., Mwakigonja A.R., et al. Lymphatic and vascular origin of Kaposi's sarcoma spindle cells during tumor development. Int J Cancer. 2006; 119(6):1262-7 